\title{
The influence of pressure sum, fiber blend ratio, and basis weight on wet strength and dispersibility of wood pulp/Lyocell wetlaid/ spunlace nonwovens
}

\author{
Yinjiang Zhang ${ }^{1} \cdot$ Xiangyu Jin ${ }^{1}$
}

Received: 2 November 2017 / Accepted: 26 December 2017 / Published online: 2 February 2018

(c) The Japan Wood Research Society 2018

\begin{abstract}
Wetlaid/spunlace(wetlace) nonwovens with sufficient wet strength in use and disintegration under flow field can be widely used in dispersible moist wipes. To achieve superior properties, wood pulp/Lyocell wetlace nonwovens at different process parameters including pressure sum (sum of each Jet Head pressure), Lyocell blend ratio, and basis weight were prepared. Meanwhile, the effects of these process parameters on the wet strength and dispersibility of materials were investigated. The results show that the wet strength of wood pulp/Lyocell wetlace nonwoven increases as the increase of process parameters. Pressure sum has positive linear relationship with material wet strength and exponential relationship with material dispersibility. When pressure sum is beyond 135 bars, material basis weight has significant influence on the dispersibility. Pressure sum of 210 bars is the key value that Lyocell content affects the material dispersibility. Overall, at the minimum pressure sum of 190 bars, wood pulp/Lyocell (60/40) wetlace nonwoven $\left(80 \mathrm{~g} \mathrm{~m}^{-2}\right)$ has dispersion critical value with maximum wet strength.
\end{abstract}

Keywords Wetlaid/spunlace(wetlace) $\cdot$ Wood plup/Lyocell $\cdot$ Wet strength $\cdot$ Dispersibility

\section{Introduction}

In today's fast-spaced life, convenience has become the priority for wipe products such as moist toilette tissues, bath/ bathing wipes, and dispersible moist wipes [1]. When referring to the wiping material that requires for skin contact, spunlace nonwovens have emerged as the preferred fabrics. Spunlace fabrics comprise the largest segment of nonwovens for wipes due to their excellent properties [2]. Unfortunately, the traditional spunlace nonwoven wipes often contain fully entangled synthetic fibers and are difficult to be dispersed or degraded after being discarded into toilet, which results in adversely impacting plumbing or wastewater infrastructure.

As of now, wetlace technology for dispersible moist wipe has been drawing great attention due to sufficient wet

Yinjiang Zhang

zyjdhdx2011@hotmail.com

$\bowtie$ Xiangyu Jin jinxy@dhu.edu.cn

1 Key Laboratory of Textile Science and Technology, Ministry of Education, College of Textiles, Donghua University, No. 2999 North Renmin Road, Songjiang, Shanghai 201620, China strength in use and rapid disintegration after use [3, 4]. It is evident that wetlace method contains wetlaid and spunlace processes. Wetlaid process offers high production speed and great flexibility to achieve homogeneous paper-based products $[5,6]$. Spunlace uses fine, closely spaced, highspeed waterjets that strike a web of fibers on a drum or a belt to form spunlace nonwoven by rearranging and entangling the fibers [7]. Spunlace nonwoven with excellent properties (softness, comfortable, and high strength) can be widely applied in disposable moist wipes [8,9]. However, material wet strength and dispersibiltiy is a couple of contradictions. To maintain the balance between them, reasonable processing technology needs to be chosen.

As expected, wetlace material belongs to spunlace nonwoven. The properties of wetlace nonwoven are also determined by fiber entanglement degree [10]. As for wetlace nonwoven, many factors affect fiber entanglements and cohesions, i.e., pressure sum, fiber blend ratio, and basis weight [11]. Pressure sum represents the sum of each Jet Head pressure in spunlace process and always gives rise to the fiber entanglements. A high pressure sum has greatly potential to change the web structures and significant effect on the properties of subsequent products [7, 12]. Different fibers have different flexibility property. There is no doubt 
that different fiber blend ratios lead to different fiber entanglements and cohesions under the same waterjet pressure sum [13]. At the same time, different basis weights significantly affect the processing and production properties [14]. However, there are not reported studies for wood pulp/Lyocell wetlace nonwovens involving in these parameters.

On the other hand, Lyocell fiber derived from renewable energy sources is entirely biodegradable and has smooth surface, which prevents irritation of the skin and retains the liquid within fibers keeping the entire wipe saturated [15, 16]. Meanwhile, Lyocell fiber has highly crystalline structure in which crystalline domains are continuously dispersed along the fiber axis. This offers good dry strength as well as excellent wet strength [15]. Haeubl et al. [17] found that hydroentangled nonwovens based on Lyocell fibers offered consumers a discrete, textile-like product appearance and skin-feel. Uusi-Tarkka (bio-based nonwoven fabric-like materials produced by paper machines) used paper machines (wetlaid method) to create wood pulp/Lyocell nonwoven products. Wood pulp is a natural renewable resource that presents numerous advantages, such as high ratio of strength to weight, renewability, and processability [18]. However, wood pulp/Lyocell dispersible moist wipes from wetlace method have not been reported yet.

In this work, wood pulp/Lyocell wetlace nonwoven was prepared through different pressure sums $(80,135,190$, and 245 bars), wood pulp/Lyocell blend ratios (60/40, 70/30, and $80 / 20)$, and basis weights $\left(50,65\right.$, and $\left.80 \mathrm{~g} \mathrm{~m}^{-2}\right)$. The wood pulp/Lyocell entanglement properties of wetlace nonwoven were revealed by introducing the fiber relative flexural rigidity. The arithmetic mean wet strength was used for evaluating the mechanical behavior of materials in use. The flush tube test took advantage of stable flow shear field produced by regular rotation of tube to disintegrate the fiber aggregation, and was chosen to monitor the dispersibility of materials. Meanwhile, the influences of these varied parameters (pressure sum, fiber blend ratio, and basis weight) on wet strength and dispersiblity of materials were systematically investigated, which guide the practical production of high value (wet strength and dispersibility) wood pulp/Lyocell wetlace nonwoven for dispersible moist wipes.

\section{Materials and methods}

\section{Preparation of wetlace nonwovens}

Lyocell fiber was kindly supplied by Lenzing AG-Austria. The linear density and length of fiber were 1.7 dtex and $12 \mathrm{~mm}$, respectively. Wood pulp (KAMLOOPS KRAFT) was supplied by Domtar, Quebec City, Canada. The average width of wood pulp fibers was $36 \mu \mathrm{m}$, and the average length and thickness were $2.2 \mathrm{~mm}$ and $1.08 \mu \mathrm{m}$, respectively.
Before production, the moisture regains of wood pulp and Danufil were respectively measured, and then, the raw materials (wood pulp and Lyocell) were prepared according to the required blend ratios (60/40, 70/30, and 80/20). Regarding the pulping, the beating consistency was controlled at $3-5 \%$, the pressure difference between inlet and outlet of disc mill was set at $0.1 \mathrm{MPa}$, and the beating degree was controlled at $12.5^{\circ}-15.5^{\circ} \mathrm{SR}$. Meanwhile, Lyocell fibers were evenly dispersed in water. Next, wood pulp and Lyocell were blended to form slurry concentration, which needed to be controlled at $0-0.08 \%$. The homogenous wetlaid nonwovens with different basis weights were formed in the wet machine by the distributor, as illustrated in Fig. 1a. The production speed was kept constant at $180 \mathrm{~m} \mathrm{~min}^{-1}$. Table 1 shows the parameters of nine types of wetlaid nonwovens with different fiber blend ratios and basis weights.

In the spunlace process, it had five manifolds labeled at jet heads 1, 2, 3, 4, and 5 ( $\mathrm{JH} 1, \mathrm{JH} 2, \mathrm{JH} 3$, JH4 and JH5), as shown in Fig. 1a. Figure 1b showed the change of wetlaid nonwoven structure after spunlace process. For all the samples, $\mathrm{JH} 1$ and $\mathrm{JH} 2$ pressures both were kept constant at 40 bars. While it used the $\mathrm{JH} 3$, JH4, or JH5, each manifold was operated at the same waterjet pressure of 55 bars. In addition, each wetlaid nonwoven was respectively strengthened with four schemes of waterjet pressure sums $(80,135,190$, and 245 bars), which are listed in Table 2. The jet strips fitted into the jet heads had 24 nozzles $\cdot \mathrm{cm}^{-1}$ of $0.12 \mathrm{~mm}$ in diameter.

\section{Properties testing and characterization}

Morphologies of fibers and wetlace nonwovens were implemented on a SEM (TM3000, Hitachi, Japan). The wet strength of wetlace nonwovens was measured using YG026MB-250 testing machine (Fangyuan Textile Ins, China). Before test, materials needed to be pre-wetted by water spraying to mimic moist wipes, resulting in the water content of material was three times that of material dry weight [3]. For each sample, the experiment was repeated for five times according to the ISO 9073-3:1989 strip test method [19]. The arithmetic mean wet strength $B$ was defined as the average value of machine direction (MD) and cross-machine direction (CD) of wet strength, which could be calculated as follows (1):

$B=(\mathrm{MD}+\mathrm{CD}) / 2[N / 50 \mathrm{~mm}]$

The dispersibility or physical breakup of material was quickly measured and assessed by flush tube test developed by Trutzschler from Germany [20], as presented in Fig. 2 . Figure $2 \mathrm{a}$ shows the flush tube device for experimental start state, and Fig. $2 b$ presents the material which is completely dispersed after a certain number of rotations. Figure $2 \mathrm{c}$ 


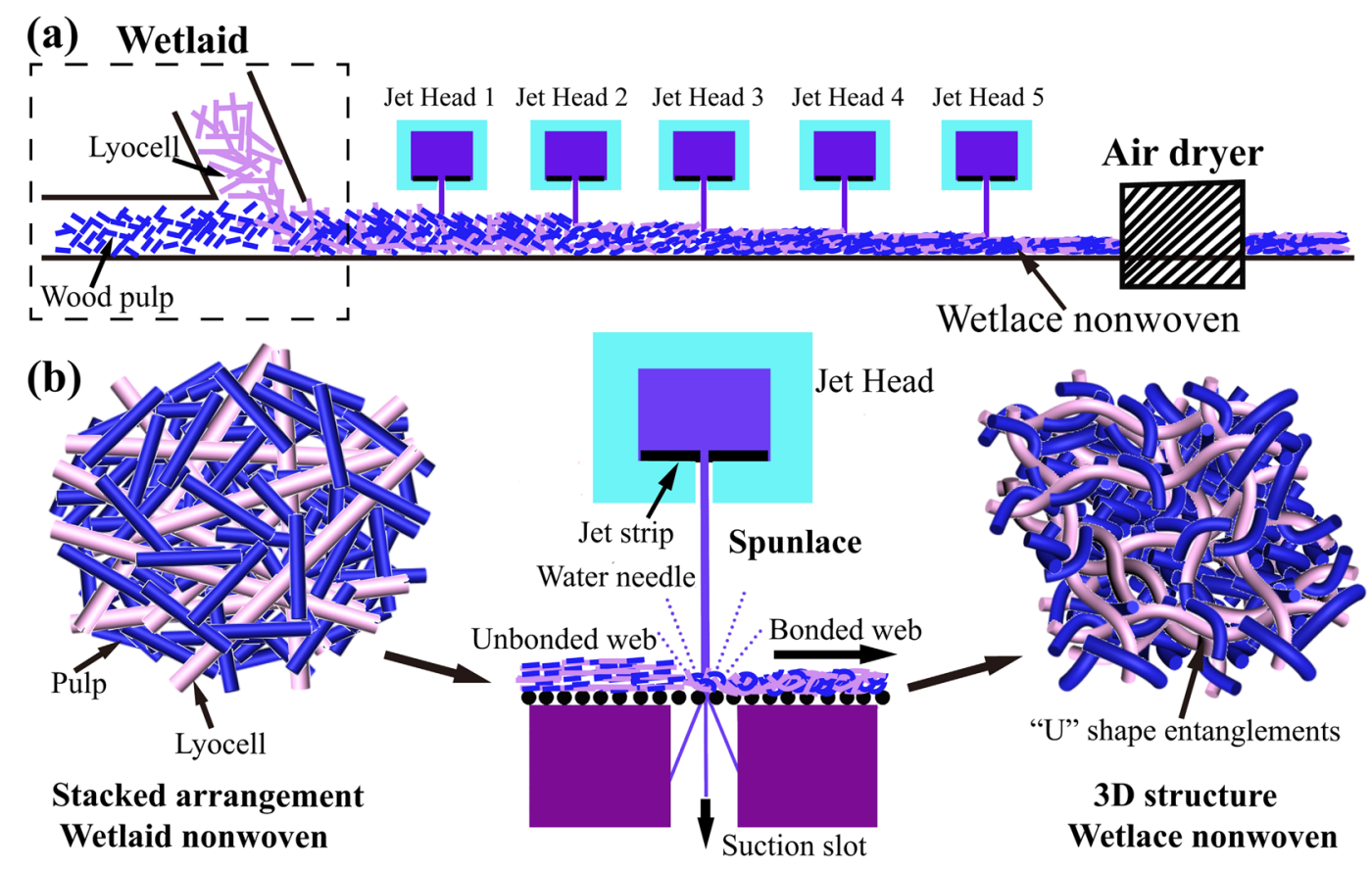

Fig. 1 Schematic diagrams of a wetlace process and $\mathbf{b}$ spunlace

Table 1 Parameters of wetlaid nonwovens

\begin{tabular}{lll}
\hline Samples & $\begin{array}{l}\text { Fiber blend ratios (wood pulp/ } \\
\text { Lyocell) }\end{array}$ & $\begin{array}{l}\text { Basis } \\
\text { weight } \\
\left(\mathrm{g} \mathrm{m}^{-2}\right)\end{array}$ \\
\hline S1 & $60 / 40$ & 50 \\
S2 & $70 / 30$ & 50 \\
S3 & $80 / 20$ & 50 \\
S4 & $60 / 40$ & 65 \\
S5 & $70 / 30$ & 65 \\
S6 & $80 / 20$ & 65 \\
S7 & $60 / 40$ & 80 \\
S8 & $70 / 30$ & 80 \\
S9 & $80 / 20$ & 80 \\
\hline
\end{tabular}

Table 2 Four types of waterjet pressure sum schemes

\begin{tabular}{llc}
\hline Scheme & Employed jet heads (JHs) & $\begin{array}{l}\text { Pressure } \\
\text { sum (bars) }\end{array}$ \\
\hline 1 & JH1 and JH2 & 80 \\
2 & JH1, JH2, and JH3 & 135 \\
3 & JH1, JH2, JH3, and JH4 & 190 \\
4 & JH1, JH2, JH3, JH4, and JH5 & 245 \\
\hline
\end{tabular}

exhibits the flush tube test for one circulation process. The measured sample size was $10 \times 10 \mathrm{~cm}^{2}$ and the added water in the tube was controlled at two-thirds of the total volume.
Firstly, sample was put into glass tube and then the tube was sealed. The equipment clamped the glass tube and rotated $180^{\circ}$. Second, it kept $1 \mathrm{~s}$ and then returned to the original position (calculated one circulation). Next, the just process was repeated. Finally, it recorded the flush tube cycles when sample broken into more than 25 pieces. If the recorded cycles were more than 200 , it meant that the material had no excellent dispersibility. Meanwhile for determining the dispersibility of material, each trial must be replicated five times.

Data of all experiments were expressed as the mean with standard deviation (SD). A 5\% significance level was used for all analyses. The paired $t$ test was used to analyze the correlation between pressure sum and wet strength, and between pressure sum and dispersion cycles.

\section{Results and discussion}

\section{Fiber and wetlace nonwoven structures}

As shown in Fig. 3a, Lyocell fiber has circular cross-section and smooth surface. Figure $3 \mathrm{~b}$ shows that wood pulp has flat cross-section and the surface is uneven with a few drapes along the fiber axis. After spunlace, Lyocell and wood pulp are swirled and entangled with each other. Notably, the nonwoven shown in Fig. $3 \mathrm{~d}$ is bonded at pressure sum of 245 bars and it visually displays a greater number of " $U$ " shape entanglements (red circle regions) compared to the 


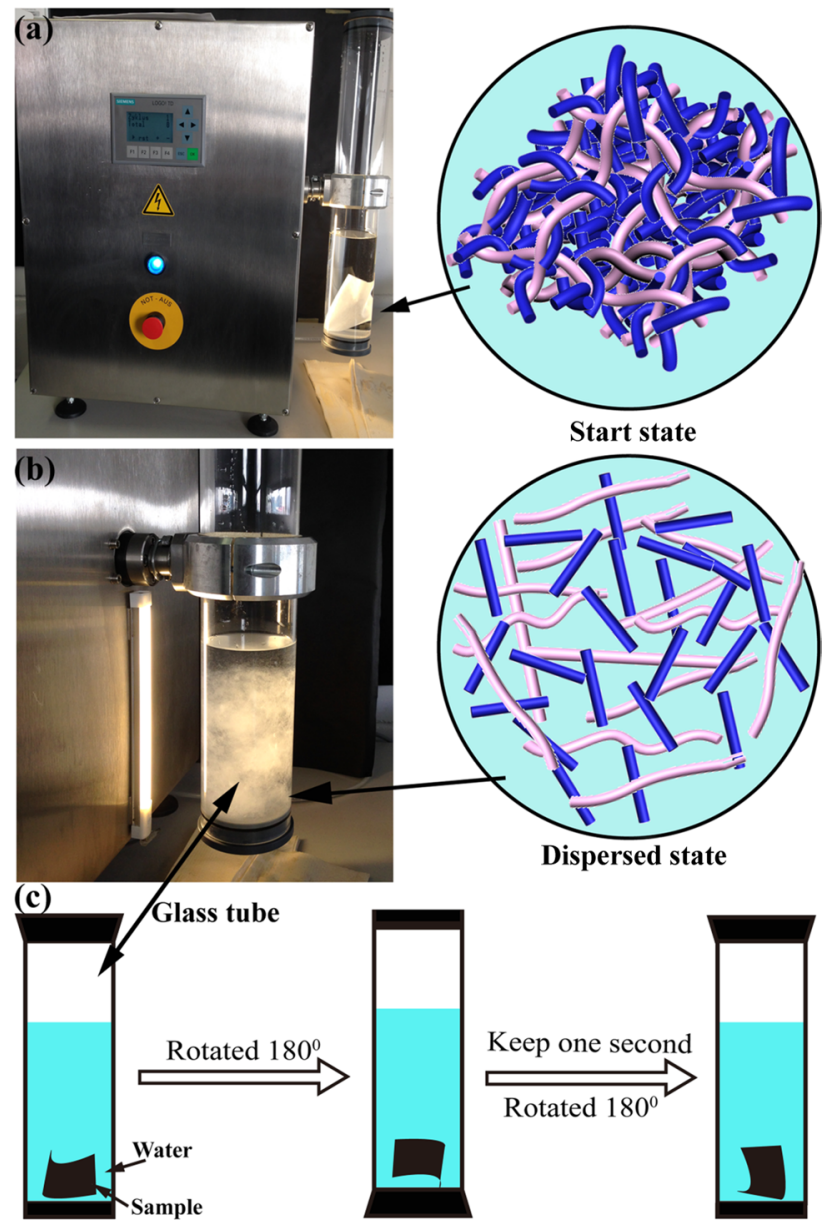

Fig. 2 Dispersion experiment of a start state, $\mathbf{b}$ dispersed state, and $\mathbf{c}$ flush tube test for one circulation

nonwoven, as shown in Fig. 3c, which is produced at pressure sum of 135 bars. It may be due to the fact that the increase in pressure sum results in increasing the fiber entanglements [7]. Meanwhile, the red dashed area (see Fig. 3d) exhibits obvious condensed ribbon-like structures (spunlace channels). However, wetlace nonwoven suffered from pressure sum of 135 bars has not obvious channel region due to low water pressure sum in manufacturing [2].

On the other hand, Mao [13] obtained that the hydroentanglement generally decreased with increasing the fiber flexural rigidity. To compare with the flexural rigidity between wood pulp and Lyocell, the fiber relative flexural rigidity based on the same linear density (1dtex) is defined as follows [21]:

$R_{\mathrm{fr}}=\frac{1}{4 \pi} \eta_{\mathrm{f}} \frac{E}{\rho} \times 10^{-5}\left[\mathrm{cN} \mathrm{cm}^{2} \mathrm{tex}^{-2}\right]$,

where $R_{\mathrm{fr}}$ is the fiber relative flexural rigidity $\left(\mathrm{cN} \mathrm{cm}^{2}\right.$ tex $\left.^{-2}\right)$, $\eta_{\mathrm{f}}$ is the cross-section shape coefficient, $\rho$ is the density of fiber $\left(\mathrm{g} \mathrm{cm}^{-3}\right)$, and $E$ is the fiber initial modulus $\left(\mathrm{cN} \mathrm{tex}^{-2}\right)$.
As the previous study, the cross-section shape coefficient of Lyocell $\left(\eta_{\text {Lyocell }}\right)$ is 0.91 [20]. Supposing that the crosssection of wood pulp is rectangular, the cross-section shape coefficient of wood pulp ( $\left.\eta_{\text {Pulp }}\right)$ can be written by the following equation:

$\eta_{\text {Pulp }}=I_{\mathrm{f}} / I_{0}=\left(b h^{3} / 12\right) /\left(d^{4} \pi / 64\right)$,

where $I_{\mathrm{f}}$ is the second moment of area of wood pulp $\left(\mathrm{cm}^{4}\right)$, $I_{0}$ is the second moment of area $\left(\mathrm{cm}^{4}\right)$ when the section is converted into the circle with same area, $b$ and $h$ are the width $(\mathrm{cm})$ and thickness $(\mathrm{cm})$ of wood pulp, respectively, and $d$ is the diameter of circle $(\mathrm{cm})$. After calculation, the average cross-section shape coefficient of wood pulp is $3.14 \times 10^{-2}$. The density of wood pulp and Lyocell is 0.7 and $1.5 \mathrm{~g} \mathrm{~cm}^{-3}$, respectively. Meanwhile, the initial modulus of Lyocell is greater than that of wood pulp $\left(E_{\text {Lyocell }}>E_{\text {Pulp }}\right)$. Then, the cross-section shape coefficient, density, and initial modulus of fibers are substituted into the Eq. (2). The relative flexural rigidity of wood pulp and Lyocell is $4.8 \times 10^{-2}$ $E_{\text {Lyocell }}$ and $3.6 \times 10^{-3} E_{\text {Pulp }}$, respectively. The result illustrates that wood pulp has small relative flexural rigidity and can be entangled easily compared to Lyocell. However, its length is too short to form a lot of fiber entanglement knots. In addition, Lyocell fiber can form the effective consolidation areas as long as it suffers from sufficient pressure sum. Therefore, Lyocell fibers are entangled into each other to form three-dimensional (3D) skeleton structure via a great number of " $U$ " shape entanglements, and wood pulp are entangled into this structure (see Fig. 3a, b), which is consistent with the previous study [11].

\section{Material wet strength}

As can be seen in Fig. 4, it displays the relationship between pressure sums and wet strength with different basis weights and Lyocell blend ratios. It is noticeable that the wet strength of wetlace nonwovens generally increases with increasing applied pressure sums. As mentioned previously, Lyocell constitutes the material skeleton of wood pulp/Lyocell wetlace nonwoven. Accordingly, in the process of wet stretching, disentanglement or slippage of Lyocell entanglement skeleton results in material fracture failure. Figure 5 shows the partial model of Lyocell fiber entanglements based on " $U$ " shape. Suppose that the fiber $I$ is regarded as Lyocell fiber, waterjet pressure sum makes the fiber $I$ entangled with $n$ root fibers. Thus, the cohesive fiber aggregations have a force on the displacement of fiber $I$. Meanwhile, discussion on the basis of " $U$ " shape entanglement is involved in several previous references $[22,23]$. Therefore, it can be concluded from the following equations (4): 
Fig. 3 SEM images of a Lyocell and $\mathbf{b}$ wood pulp, and wetlace samples produced at pressure sums of $\mathbf{c} 135$ bars and $\mathbf{d} 245$ bars

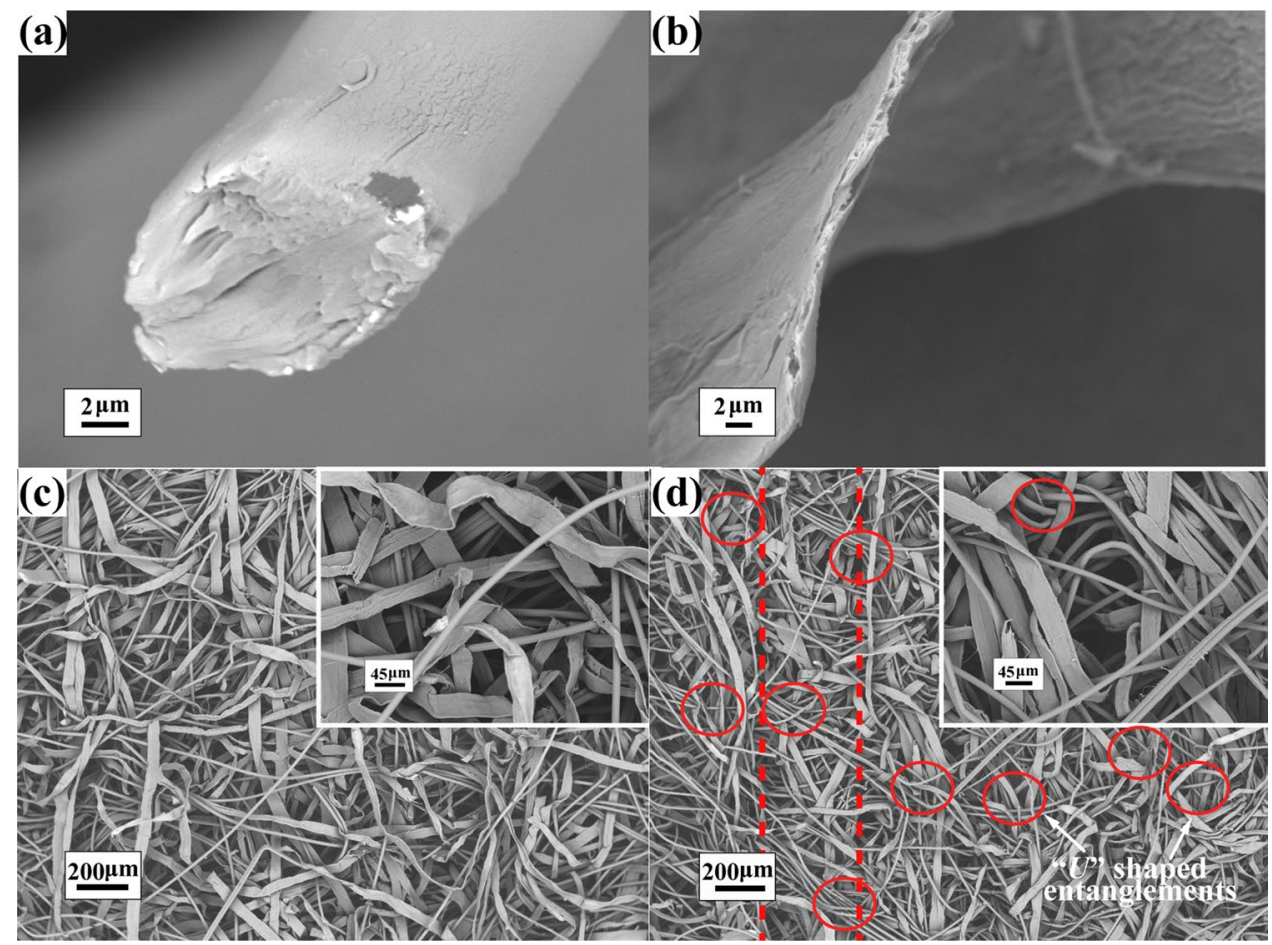

$F_{1}=F_{0} e^{\mu \theta_{0}}, F_{2}=F_{1} e^{\mu \theta_{1}}, \ldots F_{n}=F_{n-1} e^{\mu \theta_{n-1}}$,

where $\mu$ is a constant, $1,2,3,4,5 \ldots n$ is the entangled fiber around the fiber $I, F_{0}$ is the original external tension, $F_{1}$ is pull-out force that the fiber $I$ is drawn out from the basic " $U$ ", shaped entanglement (fiber $I$, fiber 1 ), and $\theta_{0}$ is the wrapped angle between fiber $I$ and fiber (1) $F_{2}$ is pull-out force that the fiber $I$ is drawn out from the fiber entanglements (fiber $I$, fiber 1 and fiber 2) and $\theta_{1}$ is the wrapped angle between fiber $I$ and fiber (2) $F_{n}$ is the pull-out force that fiber $I$ pulls from the fibrous assemblies (fiber $I$, fiber 1 , fiber 2 ... fiber $n$ ) and $\theta_{n-1}$ is the wrapped angle between fiber $I$ and fiber $n$. Therefore, the $n$ equations are summarized and Eq. (5) is obtained as follows:

$F_{n}=F_{0} e^{\mu \sum \theta_{i}}, \quad i \in(0,1,2,3 \ldots n-1)$,

where $\sum \theta_{i}$ is the sums of wrapped angles between fiber $I$ and $n$ root fibers, and mainly determines that fiber $I$ pulls out from fiber aggregations ( $n$ root fiber entanglements) in wet state. In the course of wet stretching, there are many entangled fibers like fiber $I$ that block the displacement of the fiber aggregates. Therefore, the increase in pressure sum results in increasing Lyocell fiber entanglements as well as the wrapped angles. Moreover, higher wrapped angles lead to increase the tensile strength of fiber web in wet state.

At the same time, the straight lines in Fig. 4 are the linear fit lines between the simulated and experimental results from different Lyocell contents and basis weights, which have highly linear correlation. It is also found that the wet strength of wetlace nonwovens associated with the same pressure sum can be listed as $\mathrm{S} 1>\mathrm{S} 2>\mathrm{S} 3, \mathrm{~S} 4>\mathrm{S} 5>\mathrm{S} 6$, and $\mathrm{S} 7>\mathrm{S} 8>\mathrm{S} 9$. It is reasonable to conclude that an increase in Lyocell content results in the increase of the sums of wrapped angles as well as wet strength.

In addition, comparing the wet strengths of wood pulp/ Lyocell wetlace nonwoven with different basis weights (see Fig. $4 a-c)$, we also find that under the same fiber blend ratios, the sequence of material wet strength distribution is $\mathrm{S} 7>\mathrm{S} 4>\mathrm{S} 1, \mathrm{~S} 8>\mathrm{S} 5>\mathrm{S} 2$, and $\mathrm{S} 9>\mathrm{S} 6>\mathrm{S} 3$. It is evident that the increase in nonwoven basis weight leads to increasing the material wet strength, which is consistent with the study of previous reports [8]. Because increasing the material basis weight results in increasing the Lyocell and wood pulp quantities.

Furthermore, according to the dispersible moist wipe material available in the market, the wet strength of material in use is more than $4.5 \mathrm{~N} / 50 \mathrm{~mm}$ [3]. Thus, wood pulp/ Lyocell wetlace nonwovens have sufficient wet strength in use, except wood pulp/ Lyocell (80/20, 70/30) samples under the pressure sum of 135 bars.

\section{Material dispersibility}

Figure 6 indicates the results of wetlace nonwovens dispersion at different pressure sums, fiber blend ratios, and basis weights. It is noticeable that an increase in applied pressure sum results in the increase of flush tube cycles and 

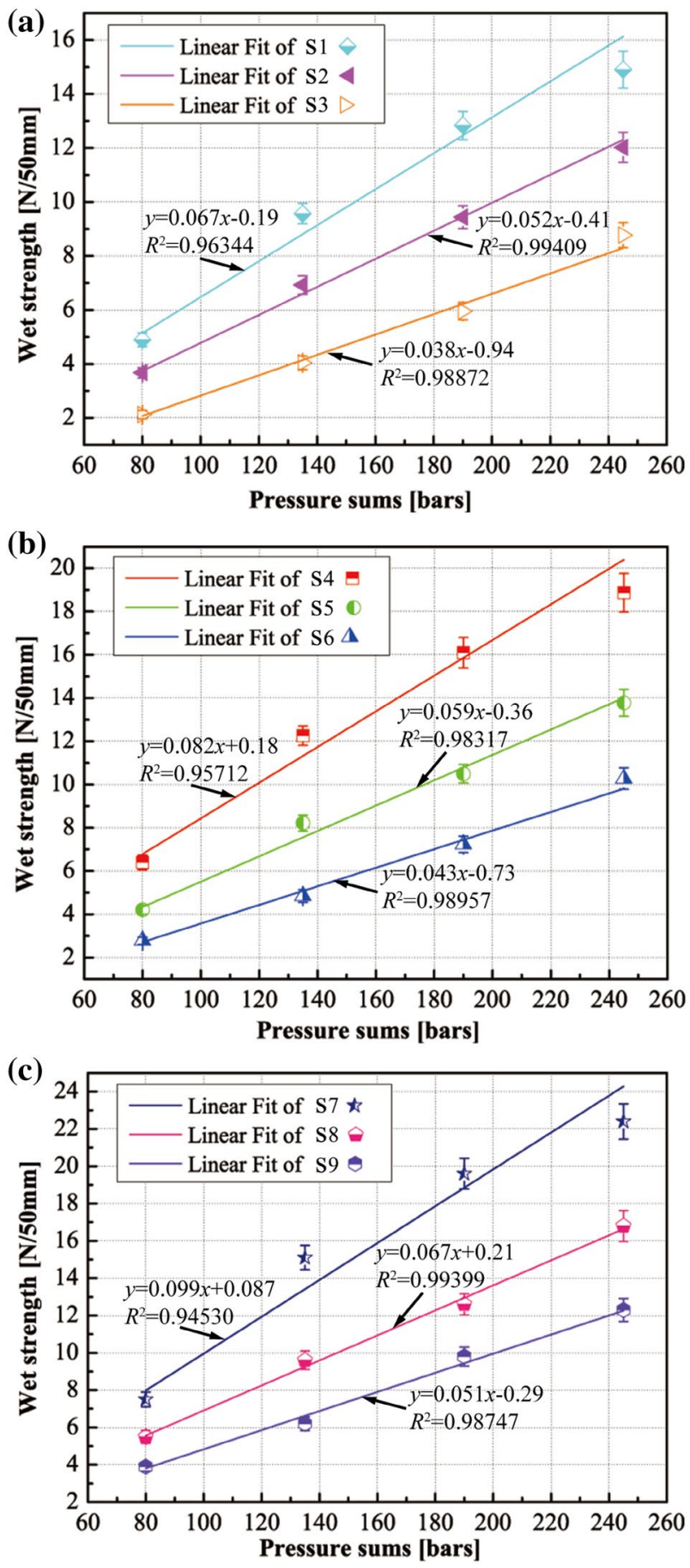

Fig. 4 Relationship between pressure sums and wet strength of a 50 , b 65 , and $\mathbf{c} 80 \mathrm{~g} \mathrm{~m}^{-2}$. Date are expressed as the mean \pm standard error, $N=5, * P<0.05$ as determined using the paired $t$ test

decrease of material dispersibility. The flush tube rotates to yield shear stress field to overcome the fiber entanglements. Pressure sum is conducive to fiber entanglements and an increase in fiber entanglements results in increasing the required shear time of water flow field as well as flush tube cycles $[24,25]$. The relationship between pressure sums and

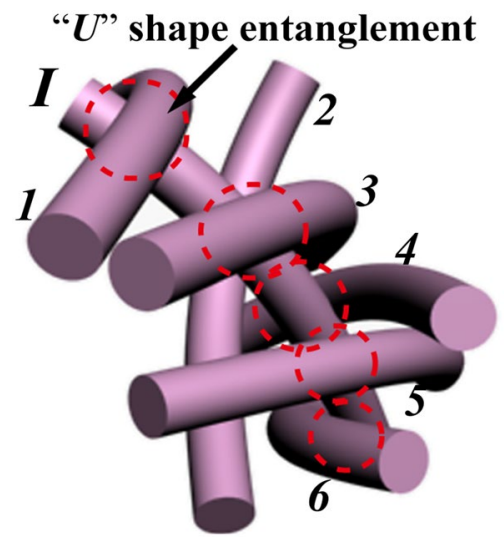

Fig. 5 Model of Lyocell fiber entanglements

dispersion cycles can be described by exponential function and have the high correlation degrees of the nonlinear fitting, as also presented in Fig. 6 .

Meanwhile, wood plup/Lyocell wetlace nonwovens have similar dispersion cycles at the pressure sum of 80 bars in spite of different fiber blend ratios and basis weights. Because small pressure sum only makes the material, surface fibers possess certain entanglements and cohesions. When the pressure sum is in the range of 80-210 bars, the required flush tube cycles increase gently. In this stage, an increase in Lyocell content leads to decrease the flush tube cycles and enhance the dispersibility. This may due to the fact that pressure sum is rather too low to achieve sufficient Lyocell entanglements in these nonwovens, especially internal fibers. While the pressure sum ranges from 210 to 245 bars, the cycles for material dispersion increase sharply and an increase in Lyocell content results in increasing the dispersion cycles as a result of sufficient Lyocell fiber entanglements of material. There is no doubt that the pressure sum of 210 bars is the key value that Lyocell content significantly affects the material dispersibility.

On the other hand, basis weight has little effect on the required dispersion cycles of materials under the low pressure sums ( $80-135$ bars). On the contrary, material basis weight has significant influence on the required dispersion cycles with high pressure sums (135-245 bars). In addition, the increase in material basis weight leads to increase in dispersion cycles. In addition, pressure sums around 210, 200, and 190 bars are found to be the dispersion critical values of samples $\left(50,65\right.$, and $\left.80 \mathrm{~g} \mathrm{~m}^{-2}\right)$, respectively. Above these pressure sums, the required flush tube cycles of samples are all more than 200 and these samples lose dispersibility thoroughly. According to these dispersion critical pressure sums, the corresponding maximum wet strength of materials is, respectively, $13.5 \mathrm{~N} / 50 \mathrm{~mm}$ (see Fig. 4a), $17.5 \mathrm{~N} / 50 \mathrm{~mm}$ (see Fig. 4b), and 19.0 N/50 mm (see Fig. 4c), and the wood pulp/Lyocell ratios are all 60/40. 

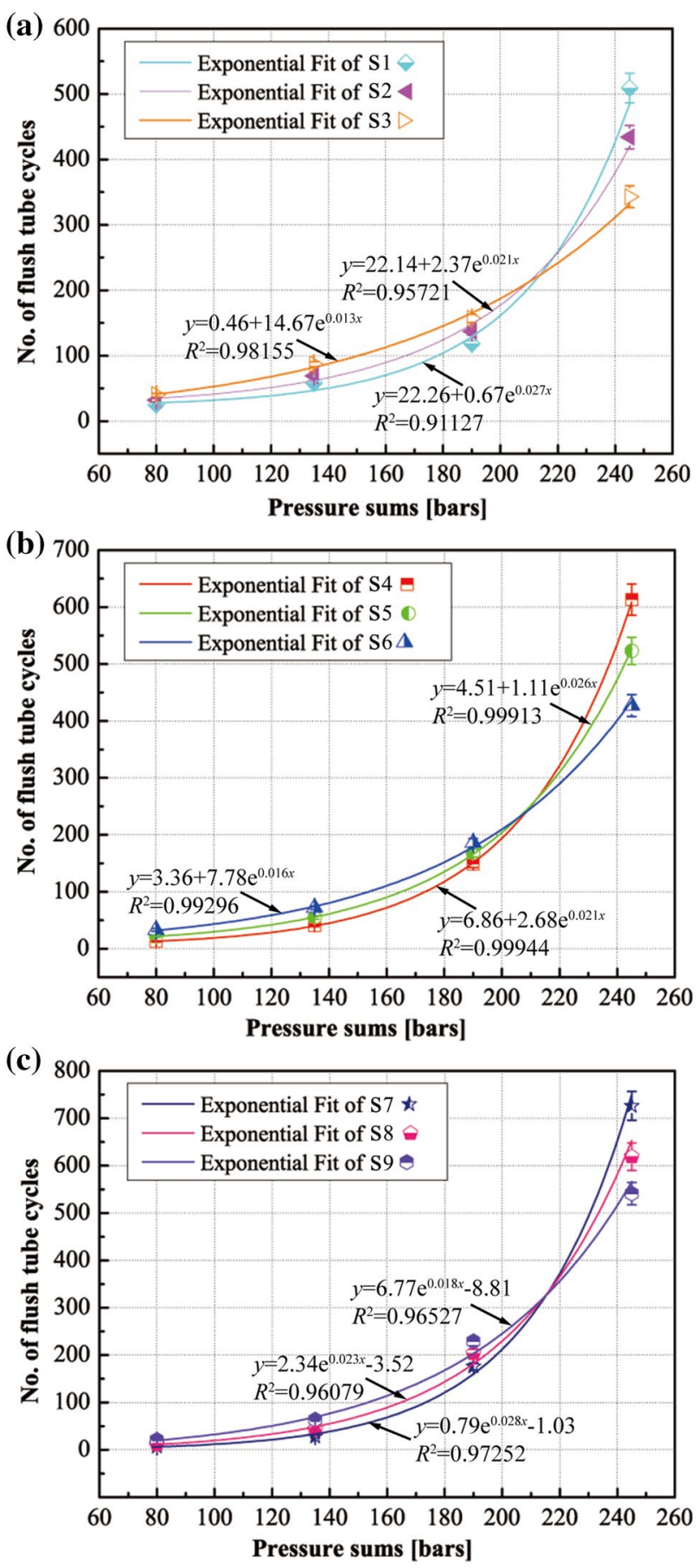

Fig. 6 Relationship between pressure sums and dispersion cycles of a 50, b 65 , and c $80 \mathrm{~g} \cdot \mathrm{m}^{-2}$. Data are expressed as the mean \pm standard error, $N=5, * P<0.05$ as determined using the paired $t$ test

As is stated above, the pressure sum, Lyocell blend ratio, and material basis weight all have significant influence on the wet strength and dispersibility of materials. At the pressure sum of 190 bars, wood pulp/Lyocell (60/40, $80 \mathrm{~g} \mathrm{~m}^{-2}$ ) wetlace nonwoven reaches the dispersion critical value and possesses the greatest wet strength compared with other samples (different basis weights and Lyocell blend ratios).

\section{Conclusions}

In this study, wood pulp/Lyocell wetlace nonwovens at different waterjet pressure sums, Lyocell blend ratios, and basis weights were fabricated, and the effects of these varied process parameters on the wet strength and dispersiblity of materials were investigated. The increase in pressure sum, Lyocell blend ratio, or basis weight results in increasing the wet strength of materials. In spite of different basis weights, materials possess similar dispersibility at the pressure sums from 80 to 135 bars. Beyond the pressure sum of 135 bars, material basis weight has significant influence on the required dispersion cycles. The increase in Lyocell content leads to decrease the flush tube cycles and the required flush tube cycles increase slowly under the pressure sum of 210 bars. But above this pressure sum, the cycles for material dispersion increase sharply and the increase in Lyocell content results in increasing the dispersion cycles. Meanwhile, pressure sums have positive linear relationship with material wet strength and exponential relationship with material dispersibility. In short, wood pulp/Lyocell (60/40) wetlace nonwoven $\left(80 \mathrm{~g} \mathrm{~m}^{-2}\right)$ has dispersion critical value with maximum wet strength in use around the smallest pressure sum of 190 bars. By control of pressure sum, fiber blend ratio, and basis weight, the wet strength and dispersibility of wood pulp/Lyocell wetlace nonwoven can be regulated and optimized.

Acknowledgements This work was supported by the Fundamental Research Funds for the Central Universities (Grant Number CUSFDH-D-2016017). The author(s) wish to express their sincere gratitude to Zhejiang Hezhong Nonwoven Co., Ltd. for their support in providing the means for conducting experiments.

\section{References}

1. Mango P (2004) Flushable wipes-past, present and future. Nonwovens Ind 2004:64-71

2. Nam S, Slopek R, Wolf D, Warnock M, Condon BD, Sawhney P, Gbur E, Reynold M, Allen C (2016) Comparison of biodegradation of low-weight hydroentangled raw cotton nonwoven fabric and that of commonly used disposable nonwoven fabrics in aerobic Captina silt loam soil. Text Res J 86:155-166

3. Zwick KJ, Vogel NJ, Baker JK (2015) Dispersible moist wipe. Patent 063637AI, US

4. Dutkiewicz JK, Fong B (2016) Dispersible nonwoven wipe material. Patent 9439549B2, US

5. Hubbe MA, Koukoulas AA (2016) Wet-laid nonwovens manufacture-chemical approaches using synthetic and cellulosic fibers. Bioresources 11:5500-5552 
6. Vaidya N, Pourdeyhimi B, Shiffler D (2003) The manufacturing of wet-laid hydroentangled glass fiber composites: preliminary results. Int Nonwovens J 2003:55-59

7. Suragani Venu LB, Shim E, Anantharamaiah N, Pourdeyhimi B (2014) Impacts of high-speed waterjets on web structures. J Text I 105:430-443

8. Pourdeyhimi B, Minton A, Putnam M (2004) Structure-processproperty relationships in hydroentangled nonwovens-part 1: preliminary experimental observations. Int Nonwovens J 13:15-21

9. Kim MO, Park TY (2016) The manufacture and physical properties of Hanji composite nonwovens utilizing the hydroentanglement process. Fiber Polym 17:932-939

10. Xiang P, Kuznetsov AV, Seyam AM (2006) Modeling of the hydroentanglement process. J Eng Fiber Fabr 1:1-15

11. Zhang Y, Zhao Y, Latifi M, Wang R, Jin X (2017) Investigation of the mechanical and dispersible properties of wood pulp/Danufil wetlaid nonwovens with/without hydroentanglement. J Text I. https://doi.org/10.1080/00405000.2017.1362747

12. Mao N, Russell SJ (2005) Structure-process-property relationship of hydroentangled fabrics. Lenzing Ber 84:50-61

13. Mao N, Russell SJ (2006) A framework for determining the bonding intensity in hydroentangled nonwoven fabrics. Compos Sci Technol 66:80-91

14. Pourmohammadi A, Russell SJ, Hoffele S (2003) Effect of water jet pressure profile and initial web geometry on the physical properties of composite hydroentangled fabrics. Text Res J 73:503-508

15. Borbély É (2008) Lyocell, the new generation of regenerated cellulose. Acta Polytechnol Hung 5(3):11-18

16. Lou C, Lin C, Chen Y, Yao C, Lin Z, Yu C, Lin J (2008) Properties evaluation of Tencel/cotton nonwoven fabric coated with chitosan for wound dressing. Text Res J 78:248-253
17. Haeubl M, Holzleithner G, Rahbaran S, Klinger J, Stanger E, Wolf C, Dvorzak M (2017) Comfort meets functionality: skin-friendly solutions for adult incontinence. Lenzing Ber 93:53-63

18. Bao M, Huang X, Jiang M, Yu W, Yu Y (2017) Effect of thermohydro-mechanical densification on microstructure and properties of poplar wood (Populus tomentosa). J Wood Sci 63:591-605

19. ISO, 9073-7 (1998). Textiles-test methods for nonwovens-Part 7: determination of bending length. International Organisation for Standarsation, London

20. Industrial Fabrics Association International (2014) Trützschler and Voith awarded quality certificate. http://advancedtextilessour ce.com/2014/01/11/trutzschlerZ-and- voith-awarded-quality-certi ficate/. Accessed 30 Oct 2017

21. Yu W, Chu C (2002) Textile physics. Donghua University Press, Shanghai, pp 2-25

22. Wang H, Pang L, Jin X, Yin B, Wu H (2010) The influences of hydrophilic finishing of PET fibers on the properties of hydroentangled nonwoven fabrics. J Eng Fiber Fabr 5:26-32

23. Xiang P, Kuznetsov AV (2008) Simulation of shape dynamics of a long flexible fiber in a turbulent flow in the hydroentanglement process. Int Commun Heat Mass 35:529-534

24. Latifi M, Tafreshi HV, Pourdeyhimi B (2008) A note on an optical method to evaluate fiber dispersion in wet-laid nonwoven process. Text Res J 78:518-523

25. Safavi A, Fathi S, Babaei MR, Mansoori Z, Latifi M (2009) Experimental and numerical analysis of fiber characteristics effects on fiber dispersion for wet-laid nonwoven. Fiber Polym $10: 231-236$ 